\title{
Bipedal Pattern Generator with Reactive Stepping based on Minimal Energy Control in the CoM State Space
}

\author{
Carlos SANTACRUZ* and Yoshihiko NAKAMURA** \\ *University of Tokyo, carlos@ynl.t.u-tokyo.ac.jp \\ **University of Tokyo, nakamura@ynl.t.u-tokyo.ac.jp
}

\begin{abstract}
We present a framework for handling disturbances in a humanoid robot during walking motion. Based on the simplified dynamics of a mass concentrated model, we adopt a two level control strategy based on the actual CoM state space. The switching criteria is determined by the extreme values of the computed ZMP. If the extreme values are within the safety limit, the disturbance can be locally compensated by modifying the trajectory to the goal state. In the other case, the final state is modified. The proposed adaptive control is based on our previously presented walking motion generation scheme based on minimal energy control.
\end{abstract}

Key Words: Locomotion, Biped robot control, Minimal energy control

\section{Introduction}

In the recent years, many algorithms to control humanoids robots with position or force based interfaces have been proposed. However, most of the methods depend on highly controlled environments. For humanoid robots with joint position control interfaces, the problem is more critical due to the impacts and collisions with the environment. A contact model cannot predict accurately the state after the moment of the impact. To compensate this factor and other unmodeled dynamics, this type of robots depend on a ZMP stabilizer which uses force-torque feedback from six-axis force sensors located on each foot. Taking into account the actual state increases the robustness against perturbations.

A common approach is to generate the original trajectory by preview control [1]. The method has been improved with pre-computation of the optimal gains [2], direct inclusion of the inequality constraint [3], inclusion of foot placements on the cost function [4] [5], and modification of the planned ZMP trajectory [6]. With optimal control based generators it is possible to adapt the trajectory based on state feedback. It is a desired condition as it increases the robustness against disturbances. These generators use a discrete linear model of the robot, most of them track an exact reference ZMP and to increase the performance the ZMP of the exact model has to be computed online and injected back to the system online which is a time consuming process.

The authors proposed an optimal based approach based on minimal energy control theory [7]. Analytical solutions of the optimal trajectories enable fast computation to achieve the desired state transfers. In this paper we propose to include CoM state feedback in the generator to regenerate the trajectory on each sample time. Depending on the current CoM state of the robot, two different control strategies guarantee physical feasibility of the trajectory. The dual strategy is based on modification of the ZMP and the final CoM state.

In Section 2. we explain the basic principles of the walking motion generation based on minimal energy control. In Section 3. we enhance the method by including the current $\mathrm{CoM}$ state of the robot in the computation. We propose a the twolevel strategy of the biped walking controller in order to realize the given footsteps and keep stability. In Section 4. we present the simulation results of the proposed scheme.

\section{Pattern Generator based on Minimal Energy Control [7]}

Let's recall the linear dynamics of a biped robot when the inertial torque around the CoM and the vertical acceleration of the CoM are neglected [8] [9]

$$
\begin{aligned}
& \ddot{x}=w^{2}\left(x-p_{x}\right) \\
& \ddot{y}=w^{2}\left(y-p_{y}\right) \\
& w \equiv \sqrt{\frac{g}{z}} \\
& p_{x}^{\min } \leq p_{x} \leq p_{x}^{\max } \quad p_{y}^{\min } \leq p_{y} \leq p_{y}^{\max }
\end{aligned}
$$

where $r=\left(\begin{array}{lll}x & y & z\end{array}\right)^{\mathrm{T}}$ denotes the position of the CoM, $p=$ $\left(\begin{array}{lll}p_{x} & p_{y} & 0\end{array}\right)^{\mathrm{T}}$ the position of the CoP, and $w$ is the natural frequency. $p_{x}^{\min }, p_{x}^{\max }, p_{y}^{\min }$, and $p_{y}^{\max }$ are the ZMP limits given by the convex hull of supporting points.

From Eq.(1), if the state vector is composed of the position and velocity of the CoM we obtain a linear 4th order state space realization as

$$
\begin{gathered}
\left(\begin{array}{c}
\dot{x} \\
\dot{y}
\end{array}\right)=\left(\begin{array}{cc}
A & 0_{2 \times 2} \\
0_{2 \times 2} & A
\end{array}\right)\left(\begin{array}{l}
x \\
y
\end{array}\right)+\left(\begin{array}{cc}
B & 0_{2 \times 1} \\
0_{2 \times 1} & B
\end{array}\right)\left(\begin{array}{l}
p_{x} \\
p_{y}
\end{array}\right) \\
x=\left(\begin{array}{c}
x \\
v_{x}
\end{array}\right) y=\left(\begin{array}{c}
y \\
v_{y}
\end{array}\right) \\
A=\left(\begin{array}{cc}
0 & 1 \\
w^{2} & 0
\end{array}\right) B=\left(\begin{array}{c}
0 \\
-w^{2}
\end{array}\right)
\end{gathered}
$$

The system can be transfered from an initial state $\mathbf{x}_{0}$ to a final state $\mathbf{x}_{f}$ in a fixed time $T$ under the constraint given by Eq.(4). 
The minimal energy control input achieves this transfer and minimizes the cost functional

$$
\int_{0}^{T} p_{r}\left(t^{\prime}\right)^{2} d t^{\prime}
$$

From hereafter $r$ is used to reference analysis that can be applied to both the $x$ and $y$ axis. Let us assume the following notation $\mathrm{Ch}(t) \equiv \cosh (t), \operatorname{Sh}(t) \equiv \sinh (t), \operatorname{Th}(t) \equiv \tanh (t)$, $\operatorname{cTh}(t) \equiv \operatorname{coth}(t)$.

For the linear model of the biped robot, the minimal energy control is given by

$$
p_{r}(t)=\left(\begin{array}{c}
\operatorname{Sh}(w t) \\
\operatorname{Ch}(w t)
\end{array}\right)^{T} D(T)\left(r_{f}-e^{A T} r_{0}\right)
$$

where

$$
\begin{gathered}
D(T) \equiv \frac{2}{\operatorname{Sh}(w T)^{2}-(w T)^{2}} \\
\left(\begin{array}{cc}
w T \operatorname{Ch}(w T)+\operatorname{Sh}(w T) & -T \operatorname{Sh}(w T) \\
-w T \operatorname{Sh}(w T) & \frac{1(w T \operatorname{Ch}(w T)-\operatorname{Sh}(w T))}{w}
\end{array}\right) \\
e^{A T}=\left(\begin{array}{cc}
\operatorname{Ch}(w T) & w^{-1} \operatorname{Sh}(w T) \\
w \operatorname{Sh}(w T) & \operatorname{Ch}(w T)
\end{array}\right)
\end{gathered}
$$

With the computed ZMP given by Eq.(7), the analytical solution for the CoM state trajectory is

$$
\begin{aligned}
r(t) & =e^{A t} r_{0}+\int_{0}^{t} e^{A(t-s)} B p_{r}(s) d s \\
& =e^{A t} r_{0}+\frac{1}{2} S(t) D(T)\left(r_{f}-e^{A T} r_{0}\right)
\end{aligned}
$$

where

$$
S(t) \equiv\left(\begin{array}{cc}
\operatorname{Sh}(w t)-w t \operatorname{Ch}(w t) & -w t \operatorname{Sh}(w t) \\
-w^{2} t \operatorname{Sh}(w t) & \frac{(\operatorname{Sh}(w t)+w t \operatorname{Ch}(w t)}{-w}
\end{array}\right)
$$

The boundary conditions are determined from the step parameters (Fig. 1) as

$$
\begin{aligned}
\left(\begin{array}{c}
x_{0}^{i} \\
y_{0}^{i}
\end{array}\right) & =-\left(\begin{array}{c}
x_{f}^{i-1} \\
y_{f}^{i-1}
\end{array}\right) \\
\left(\begin{array}{c}
v_{0 x}^{i} \\
v_{0 y}^{i}
\end{array}\right) & =\left(\begin{array}{c}
v_{f x}^{i-1} \\
v_{f y}^{i-1}
\end{array}\right) \\
\left(\begin{array}{c}
x_{f}^{i} \\
y_{f}^{i}
\end{array}\right) & =\frac{1}{2}\left(\begin{array}{c}
L^{i} \\
(-1)^{s_{i}} W^{i}
\end{array}\right) \\
\left(\begin{array}{c}
v_{f x}^{i} \\
v_{f y}^{i}
\end{array}\right) & =\left(\begin{array}{c}
w \operatorname{cTh}\left(w T^{i} / 2\right) x_{f}^{i} \\
w \operatorname{Th}\left(w T^{i} / 2\right) y_{f}^{i}
\end{array}\right)=\left(\begin{array}{l}
w k_{v x} x_{f}^{i} \\
w k_{v y} y_{f}^{i}
\end{array}\right)
\end{aligned}
$$

Based on the boundary conditions the initial and final states are given by

$$
\begin{aligned}
r_{0}^{i} & =\left(\begin{array}{ll}
r_{0}^{i} & v_{0 r}^{i}
\end{array}\right)^{\mathrm{T}} \\
r_{f}^{i} & =\left(\begin{array}{ll}
1 & w k_{v r}
\end{array}\right)^{\mathrm{T}} r_{f}^{i}
\end{aligned}
$$

This solution doesn't guarantee that the constraint given by Eq.(4)is satisfied. However, assuming instantaneous change of support, no discontinuity in velocities due to impacts, and constant stepping time, it is possible to find limits on the step parameters in order to keep the trajectory physically consistent. Details can be found in [7].

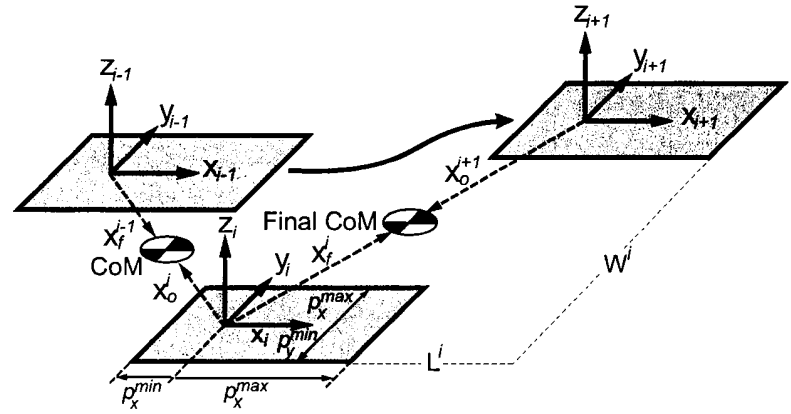

Figure 1: Step parameters

\section{Two level Control Strategy Based on the CoM State Space}

With the method explain in the previous section it is possible to generate walking motions that a ZMP based stabilizer can compensate and a humanoid robot execute. However, physical consistency is only guaranteed when the boundary conditions are strictly satisfied. Desired foot rotation in the yaw axis, changing the stepping time between steps, or disturbances during the step modify the boundary conditions. In this case it is not possible to guarantee a physically consistent motion. However, the pattern generator is based on an optimal formulation so it is possible to take into account the current state of the robot to compensate perturbations

The trajectories given by Eq.(7) Eq.(10) can be recomputed on any intermediate point. For the control law, we generate the next sample of the ZMP and CoM trajectories based on the actual state, namely

$$
\begin{aligned}
p_{r}\left(t_{s}\right) & =\left(\begin{array}{c}
\mathrm{Sh}\left(w t_{s}\right) \\
\mathrm{Ch}\left(w t_{s}\right)
\end{array}\right)^{\mathrm{T}} D\left(T_{l}\right)\left(r_{f}-e^{A T_{l}} r_{a}\right) \\
r\left(t_{s}\right) & =e^{A t_{s}} r_{a}+\frac{1}{2} S(t) D\left(T_{l}\right)\left(r_{f}-e^{A T_{l}} r_{a}\right)
\end{aligned}
$$

$r_{a}=\left[x_{a}, v_{a}\right]$ is the CoM current state of the robot, $T_{l}$ is the remaining time to desired goal, and $t_{s}$ denotes the sample time. The system is full-state reachable, therefore stability of the closed loop system is guaranteed. In case the are no disturbances, the principle of optimality guarantees that the generated trajectory will be the same as if we generated the trajectory from the initial state to the final state. The analytical solution enables fast computation and the generated trajectory is optimal.

Fig.2a shows different CoM state space and ZMP trajectories corresponding to a state transfer from $x_{0}$ to $x_{f}$ from 2 [s] to $3[\mathrm{~s}]$. The blue line corresponds to the original trajectory with no disturbance. For the other trajectories, disturbances were added to the system as instantaneous changes of velocity . Notice that for the disturbances of magnitude $0.1[\mathrm{~m} / \mathrm{s}]$, the generator modifies the CoM trajectory and ZMP input to reach the desired goal. For the disturbances of $0.2[\mathrm{~m} / \mathrm{s}]$, even if the desired final state can be reached, the trajectory is physically infeasible. The main problem is related to the control magnitude of the input as it is bounded by Eq.(4). The problem simplifies to finding the extreme values of the function. 

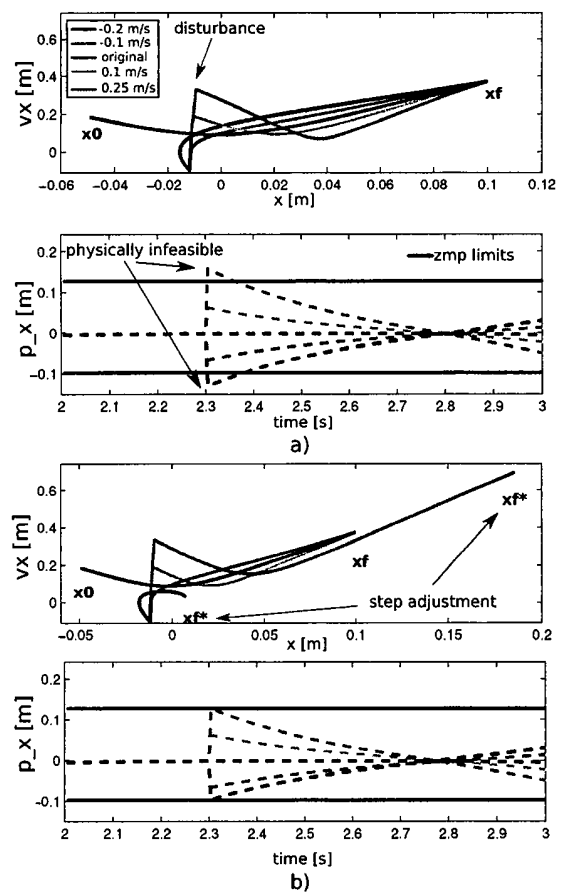

Figure 2: Generated CoM state space trajectory ( $v x$ vs $x$ ) and ZMP trajectory ( $p_{r}$ vs $t$ ) a) real-time generation based on the current state b) real-time generation with final state correction.

Due to the fact that it is either an hyperbolic sine or cosine, besides the boundary values $p_{r}\left(t_{s}\right), p_{r}\left(T_{l}\right)$ we need to find if the function has a critical point $p_{r}(t c)$. From Eq.(7), we define

$$
n_{r}\left(r_{f}, r_{0}, T\right) \equiv D(T)\left(r_{f}-e^{A T} r_{0}\right)=\left(\begin{array}{ll}
n_{r 1} & n_{r 2}
\end{array}\right)^{\mathrm{T}}
$$

Taking the derivative of $p_{r}(t)$, we find the critical point which is given by

$$
p\left(t_{c}\right)=\frac{1}{w} \operatorname{atanh}\left(-\frac{n_{r 1}}{n_{r 2}}\right) \text { if }-\operatorname{Th}\left(w T_{l}\right)<\frac{n_{r 1}}{n_{r 2}}<0
$$

On each sample time we search for the extreme values of the function from $p_{r}\left(t_{s}\right), p_{r}\left(T_{l}\right)$, and $p_{r}\left(t_{c}\right)$ if exists. If the three values are within the supporting region determined by the foot limits, the final goal is reachable and the next sample can be generated.

In case that the extreme values are outside the convex hull of supporting points, the robot will tip and foot rotation occurs. To generate a physically consistent motion with less control magnitude, it is necessary to adjust parameters. It is possible to adjust the final position of the CoM $r_{f}$, the final velocity of the CoM $v_{f}$, and the remaining step time $T_{l}$. Modifying the final velocity $v_{f}$ breaks the relationship given by Eq.(15), the remaining step time $T_{l}$ appears nonlinearly in the ZMP equations and it is not possible to find an analytical solution to the correction value. Moreover, it also affects the other axis of motion as it is a coupling term in the linear model.

We select to adjust the final position of the CoM $r_{f}$ by modifying the desired next step position as in Eq.(14). The final velocity $v_{f}$ will be modified by the relationship given in Eq.(15).
The derivative of the computed ZMP with respect to the adjustment parameter is

$$
\frac{\partial p_{r}}{\partial r_{f}}=\left(\begin{array}{l}
\operatorname{Sh}\left(w t_{s}\right) \\
\operatorname{Ch}\left(w t_{s}\right)
\end{array}\right)^{\mathrm{T}} D\left(T_{l}\right)\left(\begin{array}{c}
1 \\
w k_{v r}
\end{array}\right)
$$

It can be shown that the derivative has negative value for $t_{s}=$ 0 . It justifies the intuitive result that in case of an increase of kinetic energy a larger step should be taken in the direction of the velocity vector. If the disturbance decreases the kinetic energy, the step should be reduced in order to keep the ZMP trajectory within the supporting region. We need to consider how much should be the adjustment of the final position. It is possible to adjust the value to bring the $p_{r}(t s)$ to a zero value. However, stability and keeping the kinematic workspace of the legs are competing objectives. We find the value of $r_{f}^{*}$ that will generate that the next sample of the ZMP $p_{r}^{*}(t s)$ is in a safety limit of the supporting region. This value is obtained from Eq.(18) as

$$
r_{f}^{*}=\frac{p_{r}^{\text {limit }}+\left(\begin{array}{c}
\mathrm{Sh}\left(w t_{s}\right) \\
\mathrm{Ch}\left(w t_{s}\right)
\end{array}\right)^{\mathrm{T}} D\left(T_{l}\right) e^{A T_{l}} r_{a}}{\left(\begin{array}{c}
\mathrm{Sh}\left(w t_{s}\right) \\
\mathrm{Ch}\left(w t_{s}\right)
\end{array}\right)^{\mathrm{T}} D\left(T_{l}\right) k_{v}}
$$

where $p_{r}^{\text {limit }}$ denotes the desired position of the ZMP in the next sample time and $k_{v}=\left(\begin{array}{ll}1 & w k_{v r}\end{array}\right)^{\mathrm{T}}$ as in Eq.(17). The final state vector is modified as

$$
r_{f}^{*}=k_{v} r_{f}^{*}
$$

With this new final state, we check that the landing position of the foot is within the kinematic workspace. Fig. $2 b$ shows the CoM trajectory with the modification of the step length with the proposed step adjustment law. $p_{r}^{\text {limit }}$ was set up to $100 \%$ of the boundary of the supporting region. It can be seen that now even in the presence of strong disturbances, the ZMP value stays on the limit and a physically consistent motion is generated.

\section{Simulation results}

With the proposed control scheme we generated a trajectory for walking straight. The step length was $L^{i}=0.1[\mathrm{~m}]$, the step width $W^{i}=0.18[\mathrm{~m}]$, the step time $T^{i}=1.0[\mathrm{~s}]$, and the CoM height $h^{i}=0.8[\mathrm{~m}]$. We added disturbances in the $y$-axis of magnitude $0.05[\mathrm{~m} / \mathrm{s}]$ and $0.15[\mathrm{~m} / \mathrm{s}]$ at $1.5 \mathrm{~s}$ and $3.3 \mathrm{~s} \mathrm{re}-$ spectively. In the $x$-axis a disturbance of $0.2[\mathrm{~m} / \mathrm{s}$ ] was added at 4.4 [s].

The first impact at 1.5 [s] is compensated by recomputing the ZMP input and the final goal is reached. The situation is different for the second disturbance at 3.3 [s]. In this case the generator computes the correction value of the final state in order to keep the ZMP bounded. It causes the step width to increase with respect to the planned value. The same situation can be seen at 4.4 [s] for the $x$-axis. The motion generator increases the length of the step and stability is maintained. The generator uses the actual value to modify the trajectory 

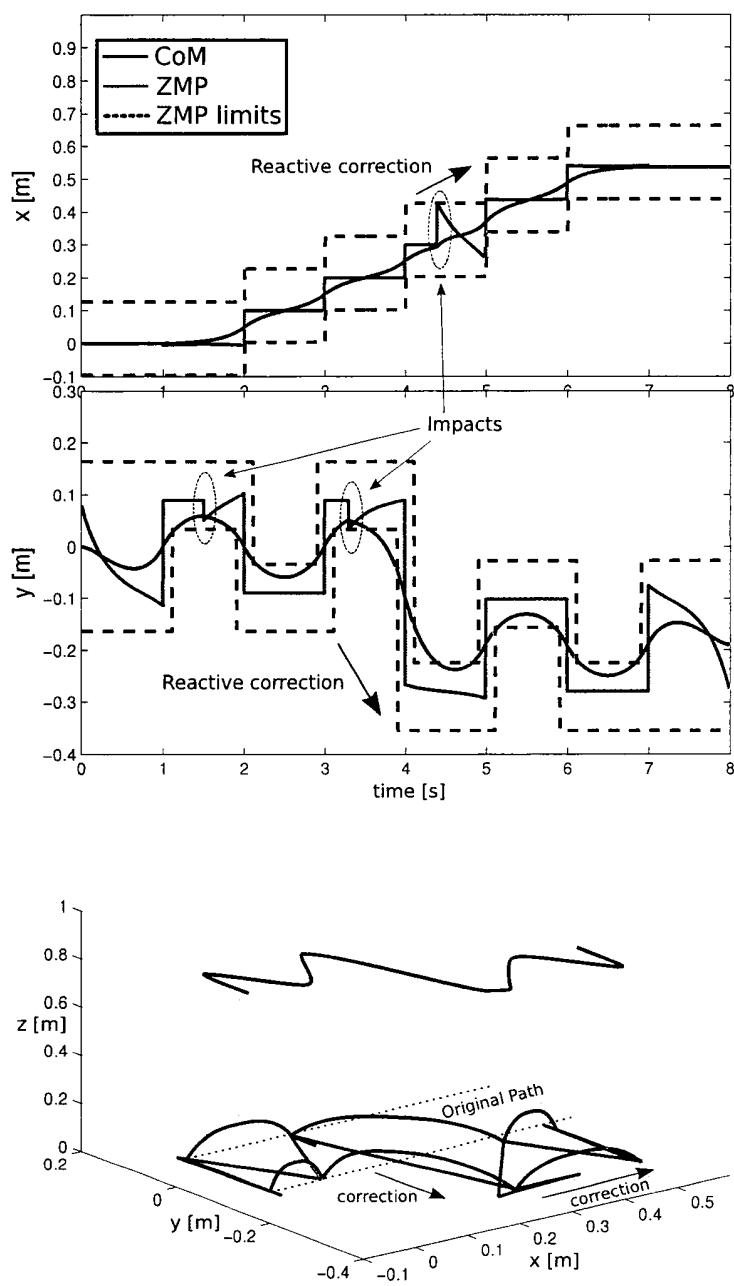

Figure 3: Control scheme and generation of CoM state trajectories with state feedback and recalculation every sample time

towards the goal. Notice there is no reference ZMP trajectory. The ZMP is computed on each step based on the actual state, the final state, and the remaining time. The generator is constantly adapting its gains in order to reach the desired final state. When the actual state generates a trajectory with computed ZMP extreme values outside the boundaries, the final state is modified.

\section{Conclusions}

We proposed a walking motion scheme that updates the trajectory based on the actual CoM state of the robot. If the disturbance is low, the trajectory is continuously modified in order to reach the desired goal position. In case of stronger disturbances, we proposed the final desired state is modified in order to generate a physically feasible motion.

The system doesn't follow a strict ZMP trajectory and it is computationally simple as all the expressions an parameters have analytical solution.

Real-time modification of the gait pattern is more robust against unknown disturbances. However, the original trajectory is not followed which could be desired in tasks such as human motion imitation.

The final state adjustment is based on modifying the desired next step's position. The kinematic workspace of the robot and the physical ZMP limits determine the maximum magnitude that can be compensated with the reactive step. In order to handle stronger disturbances, higher level control strategies such as distributing the disturbance in more steps, modifying the remaining step time, or modifying the CoM height should be considered.

For the implementation of the algorithm in the real robot, as the CoM state has to be estimated from the encoder values, impacts have to be avoided in the moment of generation.

\section{Acknowledgments}

This research was supported by the Ministry of Education, Science, Sports and Culture, Grant-in-Aid for Scientific Research (S), 2008-2012, 20220001, "Establishing Human-Machine Communication through Kinesiology and Linguistics Integration" (PI: Y. Nakamura).

\section{References}

[1] S. Kajita, F. Kanehiro, K. Kaneko, K. Fujiwara, K. Harada, K. Yokoi, and $\mathrm{H}$. Hirukawa, "Biped walking pattern generation by using preview control of zero-moment point," in IEEE Int. Conf. on Robotics and Automation, vol. 2, pp. $1620-1626,2003$.

[2] K. Nishiwaki and S. Kagami, "High frequency walking pattern generation based on preview control of zmp," in IEEE Int. Conf. on Robotics and Altomation, 2006.

[3] P.-B. Wieber, "Trajectory free linear model predictive control for stable walking in the presence of strong perturbations," in IEEE-RAS Int. Conf. on Humanoid Robots, pp. 137 - 142, 2006.

[4] H. Diedam, D. Dimitrov, P.-B. Wieber, K. Mombaur, and M. Diehl, "Online walking gait generation with adaptive foot positioning through linear model predictive control," in IEEE/RSII Int. Conf. on Intelligent robots and systems, vol. 24, pp. 1121-1126, 2008.

[5] B. Stephens and C. Atkeson, "Push recovery by stepping for humanoid robots with force controlled joints," in IEEE-RAS Int. Conf. on Humanoid Robots, pp. $52-59,2010$.

[6] K. Nishiwaki and S. Kagami, "Strategies for adjusting the zmp reference trajectory for maintaining balance in humanoid walking," in IEEE Int. Conf. on Robotics and Automation, 2010.

[7] C. Santacruz and Y. Nakamura, "Walking motion generation of humanoid robots: Connection of orbital energy trajectories via minimal energy control," in IEEE-RAS Int. Conf. on Humanoid Robots, pp. 695$700,2011$.

[8] K. Mitobe, G. Capi, and Y. Nasu, "Control of walking robots based on manipulation of the zero moment point," Robotica, vol. 18 , no. 06 , pp. 651-657, 2000.

[9] T. Sugihara, Y. Nakamura, and H. Inoue, "Real-time humanoid motion generation through $\mathrm{zmp}$ manipulation based on inverted pendulum control," in IEEE Int. Conf. on Robotics and Automation, vol. 2, pp. $1404-$ 1409, 2002.

[10] F. Kanehiro, H. Hirukawa, and S. Kajita, "Openhrp: Open architecture humanoid robotics platform," The International Journal of Robotics Research, vol. 23, no. 2, pp. 155-165, 2004.

[11] K. Kaneko, F. Kanehiro, S. Kajita, M. Morisawa, K. Fujiwara, K. Harada, and H. Hirukawa, "Slip observer for walking on a low friction floor," in IEEE/RSJ Int. Conf. on Intelligent Robots and Systems, 2005. 